\title{
A CHARACTERIZATION OF COMPACT SURFACES WITH CONSTANT MEAN CURVATURE
}

\author{
MASAAKI UMEHARA
}

(Communicated by Jonathan M. Rosenberg)

\begin{abstract}
Surfaces in a 3-space of constant curvature, whose arbitrary sufficiently small open subsets admit a non-trivial isometric deformation preserving the mean curvature function, are called locally $H$-deformable. It is well known that surfaces with constant mean curvature which are not totally umbilical are all locally $H$-deformable. Conversely, we shall show in this paper that any compact locally $H$-deformable surface has constant mean curvature.
\end{abstract}

\section{INTRODUCTION}

We denote by $M^{3}(c)$ the complete simply connected 3-manifold of constant sectional curvature $c$. It is well known that any surface with constant mean curvature in $M^{3}(c)$, which is not totally umbilical, admits locally a non-trivial isometric deformation preserving the mean curvature function. The converse is not true. Surfaces whose arbitrary sufficiently small open subsets admit such a deformation are called locally $H$-deformable. Moreover, if this local deformation can be extended globally, it is called $H$-deformable. There are $H$-deformable surfaces with non-constant mean curvature. Recently, Colares and Kenmotsu [1] classified $H$-deformable surfaces with constant sectional curvature.

But in compact case, Tribuzy [6] shows that local $H$-deformability characterizes tori with constant mean curvature in $M^{3}(c)$. In this paper, we show the following extension of Tribuzy's result for higher genus.

Theorem. Let $\Sigma$ be a compact orientable immersed surface in $M^{3}(c)$ with genus $g>0$. Then $\Sigma$ is locally $H$-deformable if and only if $\Sigma$ has the constant mean curvature.

Lawson and Tribuzy [3] show that any compact surface in $M^{3}(c)$ admits at most one other global isometric immersion with the same mean curvature function, unless its mean curvature function is constant. As we will see later, our theorem is also an improvement of their result. It should be remarked that if $g=0$ there are no $H$-deformable compact surfaces in $M^{3}(c)$. Of course

Received by the editors August 30, 1988.

1980 Mathematics Subject Classification (1985 Revision). Primary 53A10; Secondary 53C42.

Key words and phrases. Surfaces with constant mean curvature, $H$-deformation. 
many compact surfaces with constant mean curvature of higher genus in the 3sphere and the Euclidean 3-space are known ([2], [4], [5] and [7]). Our theorem characterizes many such examples.

\section{Preliminaries}

Let $x: \Sigma \rightarrow M^{3}(c)$ be an immersion of a compact oriented 2-manifold into $M^{3}(c)$. The induced metric $g$ determines a conformal structure on $\Sigma$. We shall always work in the corresponding local isothermal coordinate. With respect to such a coordinate $z=u^{1}+i u^{2}$, the induced metric can be written as

$$
g=e^{2 \omega}|d z|^{2} \text {. }
$$

Let $h=\sum_{j, k=1}^{2} h_{j k} d u^{j} d u^{k}$ be the second fundamental form of the immersion. The mean curvature $H$ is defined by

$$
H=\frac{1}{2} e^{-2 \omega}\left(h_{11}+h_{22}\right),
$$

and the $(2,0)$-component of the complexification of $2 h$, denoted by $\varphi$, is given by

$$
\varphi=\frac{1}{2}\left(h_{11}-h_{22}\right)-i h_{12} .
$$

Obviously $\Phi=\varphi d z^{2}$ is a quadratic form on $\Sigma$. Using these functions, the Gauss and the Codazzi equations are written as

$$
\begin{gathered}
|\varphi|^{2}=e^{4 \omega}\left(H^{2}-K+c\right), \\
\varphi_{\bar{z}}=e^{2 \omega} H_{z},
\end{gathered}
$$

where $\varphi_{\bar{z}}=\partial \varphi / \partial \bar{z}, H_{z}=\partial H / \partial z$ and $K$ is the Gaussian curvature of the surface.

Definition. A $H$-deformation of the immersion $x$ is a continuous map $F$ : $(-\varepsilon, \varepsilon) \times \Sigma \rightarrow M^{3}(c) \quad(\varepsilon>0)$ such that

(1) On putting $x_{t}(p)=F(t, p), x_{t}$ is an isometric immersion.

(2) $x_{0}=x$.

(3) The mean curvature function $H_{t}$ of $x_{t}$ satisfies $H_{t}=H$.

Moreover if $F$ is $C^{\infty}$, it is called a smooth $H$-deformation. An $H$ deformation is trivial if for each parameter $t$, there exists an isometry $L$ of $M^{3}(c)$ such that $x_{t}=L \circ x_{0}$. By the Gauss equation (1.4), one can easily see that $H$-deformation preserves the principal curvatures of the surface.

An isometric immersion which admits a non-trivial $H$-deformation is called $H$-deformable. An isometric immersion $x: \Sigma \rightarrow M^{3}(c)$ is called locally $H$ deformable if each point of $\Sigma$ has a neighborhood restricted to which $x$ is $H$-deformable.

For example, if $x$ has constant mean curvature, $x$ is smoothly and locally $H$-deformable unless $x$ is totally umbilical. In fact, $x$ has the constant mean 
curvature if and only if $\varphi$ is holomorphic by the Codazzi equation (1.5). For each $t \in \mathbf{R}, \varphi_{t}=\varphi e^{i t}$ and $H$ satisfy the Gauss and the Codazzi equations. By the fundamental theorem of surfaces, there exists a corresponding local $\mathrm{H}$ deformation $\left(x_{t}\right)_{t \in \mathbf{R}}$, which is non-trivial unless $\varphi$ is identically zero.

By the results of Tribuzy [6], the following assertion is obvious.

Proposition ([6]). Let $x: E \rightarrow M^{3}(c)$ be an isometric immersion of a simply connected Riemannian 2-manifold into $M^{3}(c)$. Suppose that $x$ has no umbilical point; then the following assertions are equivalent:

(1) There exist two other isometric immersions $x_{i}: \Sigma \rightarrow M^{3}(\dot{c}) \quad(i=1,2)$ such that these three immersions $x, x_{1}$ and $x_{2}$ are mutually noncongruent and have the same mean curvature function.

(2) $\Sigma$ is locally $H$-deformable.

(3) $\Sigma$ is smoothly $H$-deformable.

(4) The function $\varphi$ determined by (1.3) satisfies

$$
\Delta^{0} \log \varphi=4\left|(\log \varphi)_{\bar{z}}\right|^{2},
$$

where $\Delta^{0}=4 \partial^{2} / \partial z \partial \bar{z}$.

Remark. (1.6) is an integrability condition for the existence of a smooth $\mathrm{H}$ deformation which is constructed in the following manner: By this integrability condition, for a fixed $p \in \Sigma$ and each $s \in \mathbf{R}$, there exists a unique realvalued smooth function $\theta_{s}: \Sigma \rightarrow \mathbf{R}$ such that $\theta_{s}(p)=s$, and $\varphi_{S}=\varphi \exp \left(i \theta_{s}\right)$ satisfies the Gauss and the Codazzi equations (1.4), (1.5) with respect to the mean curvature function $H$ of $x$. By the fundamental theorem of surfaces, the corresponding smooth $H$-deformation is obtained.

\section{Proof of the theorem}

In this section, we prove the theorem stated in the introduction. We have already seen that any surface with constant mean curvature in $M^{3}(c)$ is locally $H$-deformable unless it is totally umbilical. But it is well known that a compact orientable surface with constant mean curvature in $M^{3}(c)$ is totally umbilical if and only if its genus is 0 . Hence compact surfaces with constant mean curvature of genus $g>0$ are all locally $H$-deformable. (This is also well known.)

We now prove the converse assertion. Let $\Sigma$ be a compact oriented Riemannian 2-manifold of genus $g$ and $x: \Sigma \rightarrow M^{3}(c)$ be a locally $H$-deformable isometric immersion.

Lemma 1 ([3], [6]). Umbilical points of $x$ are all isolated.

Proof. Let $p$ be an accumulate point of umbilical points. By our hypothesis there exists a non-trivial $H$-deformation $x_{t}: U \rightarrow M^{3}(c)$ on some isothermal coordinate neighborhood $(U ; z)$ at $p$. Let $\varphi_{t}$ be a function determined by (1.3) from the immersion $x_{t}$. Then by the Codazzi equation (1.5), $h_{t}=\varphi_{t}-\varphi_{0}$ 
is holomorphic on $U$. On the other hand, the Gauss equation (1.4) implies $\left|\varphi_{t}\right|=\left|\varphi_{0}\right|$. Hence umbilical points on $U$ are zeros of $h_{t}$. This implies that $\varphi_{t}=\varphi_{0}$ on $U$ and contradicts the non-triviality of $x_{t}$.

Lemma 2. Let $p \in \Sigma$ be an (isolated) umbilical point and $z=u^{1}+i u^{2}$ be an isothermal coordinate with the origin $p$. Then the function $\varphi$ determined by (1.3) from the immersion $x$ has the following Taylor expansion at $p$ :

$$
\varphi=a z^{r}+o\left(|z|^{r+1}\right)
$$

where $a \in \mathbf{C}$ and $r$ is a positive integer.

Proof. Let $x_{t}: U \rightarrow M^{3}(c)$ be a local non-trivial $H$-deformation of $x$ on an isothermal coordinate neighborhood $(U ; z)$ at $p$. Without loss of generality, we may assume that $x_{t}$ is a smooth $H$-deformation. (See Lemma 3.) Let $\varphi_{t}$ be as in the proof of Lemma 1. Since $p$ is an isolated zero of $h_{t}=\varphi_{t}-\varphi$, we can take $U$ so that $\varphi_{t} \neq \varphi$ on $U \backslash p$. Then, for each $q \in U \backslash p$, there exists a unique number $\theta_{t}(q) \in(0,2 \pi)$ such that $\varphi_{t}(q)=\varphi(q) \exp \left(i \theta_{t}(q)\right)$. Obviously $\theta_{t}$ is a smooth function on $U \backslash p$. Moreover, by taking the imaginary part of (1.6), $\theta_{t}$ is harmonic. Since $\theta_{t}$ is also bounded on $U \backslash p$, it can be smoothly extended on $U$. Now we take a sufficiently small $\varepsilon$-ball $B_{\varepsilon}(p)$ at $p$. Since $\theta_{t}(q)$ is smooth on $(-\varepsilon, \varepsilon) \times \partial B_{\varepsilon}(p)$, it is also a smooth function of $(t, q) \in(-\varepsilon, \varepsilon) \times B_{\varepsilon}(p)$. This follows immediately from the fact that any harmonic function can be determined from the boundary data by the Poisson formula.

The holomorphicity of $h_{t}$ implies

$$
\frac{\partial}{\partial \bar{z}}\left\{\varphi\left(\exp \left(i \theta_{t}\right)-1\right)\right\}=0 .
$$

Taking the derivative with respect to $t$, we have

$$
\frac{\partial}{\partial \bar{z}}\left(\dot{\theta}_{t} \varphi\right)=0
$$

where the dot means the derivative with respect to $t$. Thus $\dot{\theta}_{t} \varphi$ is also holomorphic. Suppose all derivatives of $\varphi$ vanish. Then so does $\dot{\theta}_{t} \varphi$ and hence it is identically zero. Since the $H$-deformation is non-trivial, $\dot{\theta}_{t} \neq 0$ for some $t$. Thus $\varphi$ should be identically zero, and this contradicts non-triviality of the $H$-deformation $x_{t}$. So we may put

$$
\varphi=\sum_{\alpha+\beta=r} \gamma_{\alpha \beta} z^{\alpha} \bar{z}^{\beta}+o\left(|z|^{r+1}\right),
$$

where $\sum_{\alpha+\beta=r}\left|\gamma_{\alpha \beta}\right| \neq 0$ and $r$ is a positive integer. Since $\dot{\theta}_{t}$ is a non-vanishing real-valued harmonic function, it has the Taylor expansion:

$$
\dot{\theta}_{t}=c z^{\prime}+\overline{c z}^{\prime}+o\left(|z|^{\prime+1}\right) \quad(c \neq 0) .
$$


From (2.3) and (2.4), one can easily verify that the power series expansion of $\dot{\theta}_{t} \varphi$ contains a non-vanishing term involving the variable $\bar{z}$ unless $\ell=0$. Since $\dot{\theta}_{t} \varphi$ is holomorphic, we have $\ell=0$ and $\gamma_{\alpha \beta}=0 \quad(1 \leq \beta \leq r)$. Hence

$$
\varphi=\gamma_{r, 0} z^{r}+o\left(|z|^{r+1}\right) \text {. }
$$

This proves Lemma 2.

The integer $r$ determined by the expansion (2.5) is just the winding number around a zero $p$ of the quadratic form $\Phi=\varphi d z^{2}$. Hence the sum of all such integers over umbilical points of $x$ coincides with $4(g-1)$. In particular, there are no $H$-deformable surfaces of genus 0 . So we assume $g \geq 1$ in the sequel. By our assumption, we can take a holomorphic 2-differential $\zeta$ on $\Sigma$ which is not identically zero. Let $p_{1}, \ldots, p_{n}$ be umbilical points of $x$ and $q_{1}, \ldots, q_{m}$ zeros of $\zeta$. Then the function $\log f=\log (\Phi / \zeta) \quad\left(\Phi=\varphi d z^{2}\right)$ is defined on $\Sigma \backslash\left(p_{1}, \ldots, p_{n}, q_{1}, \ldots, q_{m}\right)$ and satisfies:

$$
\begin{aligned}
\Delta^{0} \log f & =\Delta^{0} \log \varphi-\Delta^{0} \log \zeta^{0} \\
& =\Delta^{0} \log \varphi \\
& =4\left|(\log \varphi)_{\bar{z}}\right|^{2},
\end{aligned}
$$

where $\zeta=\zeta^{0} d z^{2}$. For a sufficiently small $\varepsilon>0$, we define a subset

$$
\Sigma_{\varepsilon}=\Sigma \backslash\left\{B_{\varepsilon}\left(p_{1}\right) \cup \ldots \cup B_{\varepsilon}\left(p_{m}\right) \cup B_{\varepsilon}\left(q_{1}\right) \cup \ldots \cup B_{\varepsilon}\left(q_{n}\right)\right\},
$$

and consider the integral

$$
\int_{\sum_{\epsilon}} \Delta \log f=\int_{\sum_{\epsilon}} 4 e^{-2 \omega}\left|(\log \varphi)_{\bar{z}}\right|^{2},
$$

where $\Delta=e^{-2 \omega} \cdot \Delta^{0}$ is the Laplace-Beltrami operator of $\Sigma$. Since $\Delta \log f=* d *$ $d(\log f)$, and $d(\log f)$ is independent of the choice of a branch of logarithm, we have the following by applying the Stokes formula:

$$
\begin{aligned}
\int_{\sum_{\varepsilon}} \Delta \log f= & -\sum_{j=1}^{m} \int_{\partial B_{\varepsilon}\left(p_{j}\right)} * d(\log f) \\
& -\sum_{k=1}^{n} \int_{\partial B_{\varepsilon}\left(q_{k}\right)} * d(\log f),
\end{aligned}
$$

where $*$ is the Hodge operator of $\Sigma$. On the other hand,

$$
* d \log f=i\left(\varphi_{z} d z\right) / \varphi-i\left(\varphi_{\bar{z}} d \bar{z}\right) / \varphi-i\left(\zeta_{z}^{0} d z\right) / \zeta \text {. }
$$

By Lemma 2, $\varphi$ and $\zeta^{0}$ have the following power series expansions at $p \in$ $\left\{p_{1}, \ldots, p_{m}, q_{1}, \ldots, q_{n}\right\}$ :

$$
\begin{aligned}
\varphi & =a z^{r}+o\left(|z|^{r+1}\right), \\
\zeta^{0} & =b z^{\prime}+o\left(|z|^{\gamma+1}\right) .
\end{aligned}
$$


So one can easily obtain

$$
\lim _{\varepsilon \rightarrow 0} \int_{\partial B_{\varepsilon}(p)} * d(\log f)=2 \pi i(r-\ell) .
$$

By (2.7), we have

$$
\begin{aligned}
\lim _{\varepsilon \rightarrow 0} \int_{\Sigma_{\varepsilon}} 4 e^{-2 \omega}\left|(\log \varphi)_{\bar{z}}\right|^{2} & =\lim _{\varepsilon \rightarrow 0} \int_{\Sigma_{\varepsilon}} \Delta \log f \\
& =-2 \pi\left\{\operatorname{index}(\Phi)-\operatorname{index}\left(\zeta^{0}\right)\right\} \\
& =-2 \pi\{4(g-1)-4(g-1)\}=0 .
\end{aligned}
$$

Thus $\left|(\log \varphi)_{\bar{z}}\right|^{2}=0$ and $\varphi$ is holomorphic. Now the theorem has been proven.

In the proof of the theorem, we used the following property of $H$-deformations.

Lemma 3. Let $x: \Sigma \rightarrow M^{3}(c)$ be an isometric immersion of a Riemannian 2manifold into $M^{3}(c)$. Then non-trivial $H$-deformations of $x$ are all smoothly re-parametrized.

Proof. Since a non-trivial $H$-deformation has at most one degree of freedom, we may assume that $\Sigma=U$ is a domain of $\mathbf{C}$ with the canonical coordinate $z$. A global smooth $H$-deformation is obtained from the standard continuation argument.

Let $x_{t}: U \rightarrow M^{3}(c)$ be a non-trivial continuous $H$-deformation of $x$ and $\varphi_{t}$ the function determined by (1.3) from $x_{t}$. By Lemma $1, h_{t}=\varphi_{t}-\varphi_{0}$ is holomorphic and has isolated zeros. On putting $\varphi_{t}=\exp \left(i \theta_{t}\right) \varphi_{0}$ on $U \backslash h_{t}^{-1}(0)$, as we have seen in Lemma 2, $\theta_{t}$ can be extended on $U$ as a harmonic function satisfying $0 \leq \theta_{t} \leq 2 \pi$. Then by the maximum principle, each $\theta_{t}(t \neq 0)$ cannot take the value 0 or $2 \pi$. Thus the set of umbilical points $S$ of $x_{0}$ coincides with $h_{t}^{-1}(0)$ for each $t$.

Let $p_{0} \in U \backslash S$ be a non-umbilical point. Then for $s \in \mathbf{R}$, there exists a unique real-valued smooth function $\tilde{\theta}_{s}$ on a sufficiently small neighborhood of $p_{0}$ such that $\tilde{\theta}_{s}\left(p_{0}\right)=s$ and $\varphi \exp \left(i \tilde{\theta}_{s}\right)$ satisfies the Gauss and the Codazzi equations (1.4), (1.5) with respect to the mean curvature function $H$ of $x$. (See Remark after Proposition.) Since $p_{0}$ is non-umbilical, any sufficiently small $s(|s|<\varepsilon)$ can be written as $s=\theta_{t}\left(p_{0}\right)$ and satisfies $\tilde{\theta}_{s}=\theta_{t}$. Hence $\tilde{\theta}_{s} \quad(|s|<\varepsilon)$ can be extended on $U$. By our construction, $\tilde{\theta}_{s}(q)$ is smooth on $(-\varepsilon, \varepsilon) \times U \backslash S$. Since each $\tilde{\theta}_{s}: U \rightarrow \mathbf{R}$ is harmonic and $S$ is discrete, we can conclude by using the same argument in the proof of Lemma 2 that $\tilde{\theta}_{s}(q)$ is also smooth on $(-\varepsilon, \varepsilon) \times U$. Hence the re-parametrized $H$-deformation $x_{s}$ is smooth.

Remark. Lawson and Tribuzy [3] show that any compact surface $\Sigma$ in $M^{3}(c)$ admits at most one other global isometric immersion with the same mean curvature function, unless its mean curvature function is constant. This follows 
from the theorem immediately. In fact, if there exist two other distinct isometric immersions of $\Sigma$ with the same mean curvature function, then $\Sigma$ is locally $H$-deformable by the proposition, and hence its mean curvature function is constant.

\section{REFERENCES}

1. A. G. Colares and K.Kenmotsu, Isometric deformation of surfaces in $\mathbf{R}^{3}$ preserving the mean curvature function, Pacific J. Math. 136 (1989), 71-80.

2. H. B. Lawson, Jr., Complete minimal surfaces in $S^{3}$, Ann. of Math. 92 (1970), 335-374.

3. H. B. Lawson, Jr., and R. A. Tribuzy, On the mean curvature function for compact surfaces, J. Differential Geometry 16 (1981) 179-183.

4. N. Kapouleas, Compact constant mean curvature surfaces in Euclidean three space, preprint.

5. __ Constant mean curvature surfaces in Euclidean three-space, Bull. Amer. Math. Soc., 17 (1987), 318-327.

6. R. A. Tribuzy, A characterization of tori with constant mean curvature in a space form, Bull. Soc. Brasil. Mat. 11 (1980), 259-274.

7. H. C. Wente, Counterexample to a conjecture of H. Hopf, Pacific J. of Math. 121 (1986), 193-243.

Institute of Mathematics, University of Tsukuba, Tsukuba-shi, Ibaraki 305, Japan 